\title{
A novel Automatic Defect Detection Method for Electron Beam Welded Inconel 718 components using Inductive Thermography
}

\author{
by Eider Gorostegui-Colinas*, Ander Muniategui*, Pablo López de Uralde*, Izaskun Gorosmendi*, \\ Borja Hériz*, Xabier Sabalza* \\ *IK4-LORTEK, Control and Evaluation Department, Arranomendia Kalea 4A, 20240, Ordizia, Gipuzkoa, Spain,
} egorostegui@lortek.es

\begin{abstract}
In this article Induction Thermography is applied to inspect Inconel 718 Electron Beam Welded and Tungsten Inert Gas Welded components with cracks that can go down to $180 \mu \mathrm{m}$. Satisfactory experimental results have been obtained despite the non-propitious electrical and thermal conduction properties of the material. A crack detection algorithm that uses as input phase images obtained from the FFT transformation has also been developed. It takes advantage of the rotational symmetry of the 'Butterfly-like Patterns' to convert them by using a polar Discrete Fourier Transform into 2D Gaussian like distributions that allow the identification of cracks.
\end{abstract}

\section{Introduction}

NDT inspection methods are usually based on manual techniques like Magnetic Particle or Dye Penetrant Testing, which are time consuming and user dependant. Active thermography intends to substitute these traditional methods not only to reduce inspection times, but also to provide clean and contactless inspections, something that for many industries is invaluable. Another important advantage of thermography is that this technique is robust enough to provide repeatability in the measurements and in the posterior defect detection, something that traditional inspection techniques will always lack [1] [2] [3].

In this article Induction Thermography is applied to inspect Inconel 718 Electron Beam Welded (EBW) and Tungsten Inert Gas (TIG) Welded components. The aim is to detect automatically the small cracks that may arise due to the welding process using Inductive Thermography. This is a real challenge since EBW crack sizes can go down to 180 $\mu \mathrm{m}$, which is currently at the limit of the resolution of the employed thermographic equipment. What is more, the electrical and thermal conduction properties of Inconel 718 are not in principle the most appropriate for achieving good measurements.

As stated above, apart from the experimental work performed, this research goes one step further and describes the developed algorithm to automatize the detection of the cracks. For this purpose, it is essential to note that the small sized cracks present in these components are characterized by showing a specific pattern in the extracted phase of their Fourier Transformed thermograms, commonly called Butterfly Patterns. One of the main features of this pattern is its rotational symmetry. Polar Discrete Fourier Transformation (pDFT) [4] [5] can be used to identify this type of symmetry.

\section{Induction Thermography}

There are several active thermography techniques used for non-destructive crack detection [6]. They are all based on the same fundamentals: a specimen is subjected to an external stimulation and heat propagation is captured with a thermographic camera. The propagation depends on the material's thermal properties and also on subsurface anomalies, which result in temperature differences on the surface of the considered target However, these techniques differ in the way the sample to be inspected is stimulated thermally. This excitation can be optical (with laser or flash lamps), with ultrasonic waves or by electric current. The selection of the excitation source type depends on the material of the specimen to be inspected and its defectology.

Optical stimulations have the advantage of being contactless, but depending on the sample's surface condition, emissivity and absorption coefficient, the heating can be limited and the induced thermal gradients too small to be detected by the camera. The advantage of the inductive excitation is that the heat is generated directly in the surface of the sample eliminating any heating absorption problems. In this case, a surface crack disturbs the formation of the eddy currents, deflecting the current inside the material and thereby causing an inhomogeneous heating of the surface.

As for stimulation, it can be performed harmonically or using a short pulse. In this work short pulses have been considered and then the thermal response has been evaluated using Fourier Transformation as it is explained in the following section. The major advantage of this method is that even if the crack is small (even at the limit of the equipment 
being used) the pattern appearing in the frequency spectrum (the typical butterfly shaped image) is always visible. Another advantage is that Fourier phase images are less sensitive to surface inhomogeneous heating or emissivity changes, making them more reliable. Finally, the evaluation of the thermal recording in the frequency domain allows an estimation of the depth of the defects present in the sample, since this magnitude is directly linked to the frequencies at which defects become visible [7].

One of the most important magnitudes in inductive thermography is penetration depth. It depends on the material properties (on relative permeability for example) and also on the induction frequency employed. In magnetic materials it remains very near the surface, being its depth much smaller than the depth of the crack considered. This can be emphasized by using high induction frequencies. This situation causes a direct heating of the edges of the crack and the interior of the sample to be heated mainly due to thermal diffusion. It has been shown [8] that the deeper the surface crack is, the higher is the temperature increase at the edges of the crack. In the case of non-magnetic materials on the other hand, which is the case of Inconel 718, penetration depth is comparable with the depth of the cracks present in the sample. Induced current is pushed from the edges of the crack inside the material. This causes lower current density at the edges of the cracks and after a short period of time crack tips are warmer than the edges [9].

\section{Description of Experimental Measurements}

In this work the cracks generated by the welding of Inconel 718 plates have been investigated. Two main groups are differentiated throughout the article: EBW and TIG samples. These types of welds are typical in aeronautics. This is why it is important to find an efficient way of inspection to detect possible defects that may arise during the welding process. At this stage, instead of working with whole real components, small samples with real cracks (see Fig. 1 ) have been considered to avoid unnecessary extra difficulties.

(a)

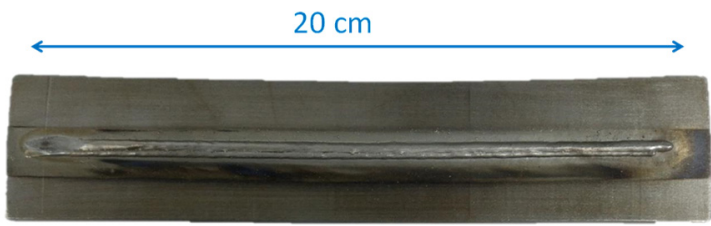

(b)

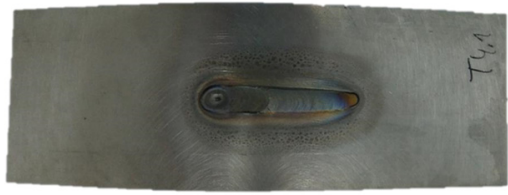

Fig. 1. Figure showing the two types of welding that have been inspected with inductive thermography with the aim of detecting surface cracks. (a) Corresponds to an EBW sample and (b) to a TIG sample. (a)

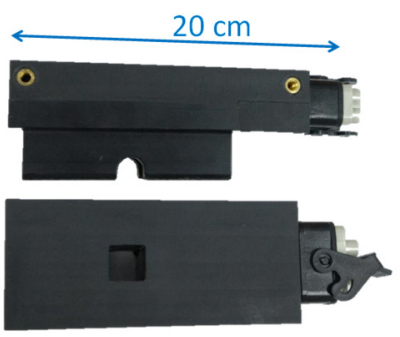

(b)

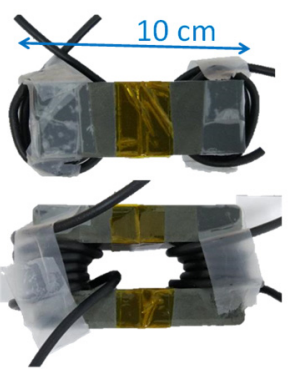

Fig. 2. Inductors employed in the inspections. a) 11, Inductor manufactured and optimized by Edevis $\mathrm{GmbH}$ (b) 12, homemade inductor still without optimization, but already giving good results.

The employed inductor generator is a mid-frequency $3 \mathrm{~kW}$ generator. As for the inductors, two models have been used depending on the sample considered. EBW samples have been excited with and inductor developed by Edevis $\mathrm{GmbH}$ [10] (hereinafter referred to as I1), since it has a geometry suitable for them. In the case of the TIG welding samples, their curvature makes difficult to position correctly inductor I1 in order to produce the correct heating. This has led to the manufacturing of a second inductor at the Research Centre itself, Inductor 12, which despite not being optimized, has provided good experimental results. Both inductors are shown in Fig. 2.

A Flir Infrared Cooled Thermal Camera has been used to perform the recordings, specifically a Flir x6541sc with the standard $50 \mathrm{~mm}$ optics and an extension ring, reaching a resolution of 45-50 $\mu \mathrm{m} / \mathrm{pixel}$. The framerate has been set to $300 \mathrm{~Hz}$ for all measurements. Finally, integration time has been set to $400-700 \mu \mathrm{s}$.

During the inspection, the sample is scanned step by step, being the inspection window $20 \mathrm{~mm} \times 20 \mathrm{~mm}$ approximately. Overlapping is always allowed in between the scans to avoid skipping any possible defects at the edges. Pulses from $100 \mathrm{~ms}$ to $200 \mathrm{~ms}$ have been employed. This interval has provided the best results together with a frequency that ranges from 10 to $35 \mathrm{KHz}$, depending on the type of sample considered. The employed setup is described schematically in Fig. 3. 


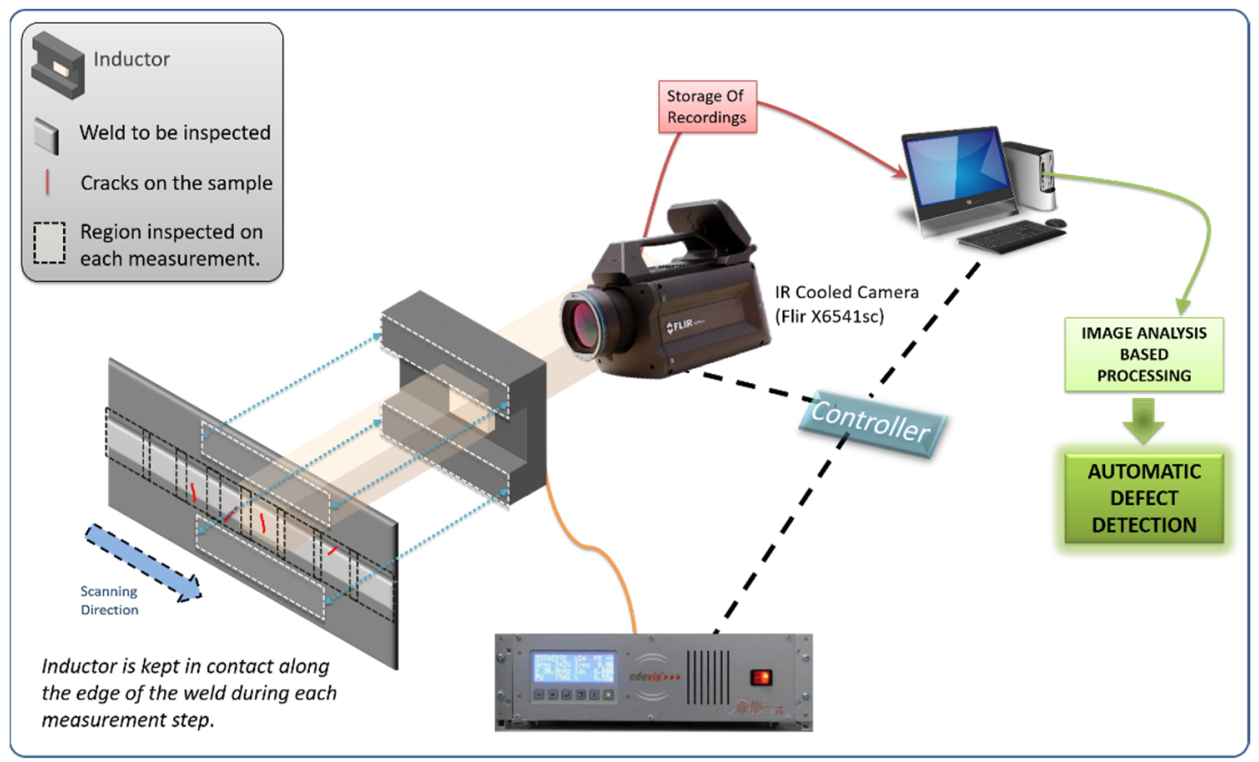

Fig. 3. Schematic figure showing the employed setup in order to inspect EWB Inconel samples using Induction Thermography.

\section{Automatic Defect Detection Algorithm Development}

In this section the stablished methodology for the automatic crack detection implementation is described. It consists of two main steps: a first processing of the recording based on pulsed phase thermography and the posterior detection.

\subsection{First Processing: FFT and the butterfly shape obtention}

As mentioned briefly before, in the present work a thermographic recording is first processed using the Fast Fourier Transform. This transformation converts time domain information into the frequency domain. The temporal content of the temperature-time trace associated with each pixel is converted to a frequency spectrum. Then, frequency components can be used to characterize defects with the advantage that phase results are independent of the surface emissivity variations in the surface of the considered sample.

Apart from that, when the employed excitation source is induction, applying the FFT is extremely useful since the phase images contain the well-known 'Butterfly Shape' at every place in the surface where a crack appears. This butterfly shape is the pattern that the posterior detection algorithm will find and locate. This is why it is important to get the best phase images as possible at this stage, making easier and reliable the application of the posterior detection algorithm.

In this regard, there are several factors affecting the quality of the Fourier phase images. On the one hand, the employed inspection parameters are critical. These parameters include the considered framerate, resolution, induction frequency and pulse time. On the other hand, the selection of the relevant recording interval for the application of the transformation is essential. In Inconel, the butterfly pattern information is mainly contained in the heating. However, it is necessary to take part of the cooling in order to obtain sharper images. What is more, when long pulses are applied, taking the whole heating is not necessary.

The other factor affecting the quality of the phase images, or more specifically the shape of the butterfly formed, is the orientation of the inductor [7] [11] [12]. A symmetric butterfly shape will only be obtained when the inductor induces currents perpendicular to the crack. This implies that the inductor must generate a magnetic field parallel to the considered crack. If a rotation between the crack and the inductor exists, the pattern still will appear, but symmetry can be lost leading to a bad posterior defect detection when the algorithm is applied. 


\subsection{Automatic Defect detection}

This section describes the developed algorithm to automatize the detection of the defects. For this purpose, it is essential to note that the small sized cracks present in these EBW components are characterized by showing a specific pattern in the extracted phase of their Fourier Transformed thermograms, commonly called Butterfly Patterns. One of the main features of this pattern is its rotational symmetry. Polar Discrete Fourier Transformation (pDFT) [4] [5] can be used to identify this type of symmetry.

The application of pDFT for Butterfly Pattern detection is described schematically in Fig. 4. Let's consider the butterfly image of Fig. 4.a, let's consider pixel $(x, y)$ as its center of symmetry and let's consider a circle of radius $r_{\text {min }}$ with center $(x, y)$. Then, lets extract the values of the pixels that lay on the perimeter of this circle and let's repeat this operation for circles of increasing radius from $r_{\min }$ to $r_{\max }$ (see Fig. 4.b). And finally, lets append all these vectors of pixel values obtained for each radius one after the other (see Fig. 4.c). The obtained extended signal will be periodical with a period of $180^{\circ}$, and hence, its FFT must show an intense peak at its corresponding equivalent frequency (see Fig. 4.d). The main differences between the cracks will be their size and orientation and these differences are expected to be appreciated in the sizes and orientation of their respective butterfly-like patterns. Their sizes can be roughly determined by adjusting the values of $r_{\min }, r_{\max }$. And their orientation can be estimated from the signal's shift owed to their inclination (see Fig. 4.e and f), i.e. the extended signal will be shifted if compared with a vertical butterfly pattern and this shift can be determined from the real and imaginary parts of the FFT.

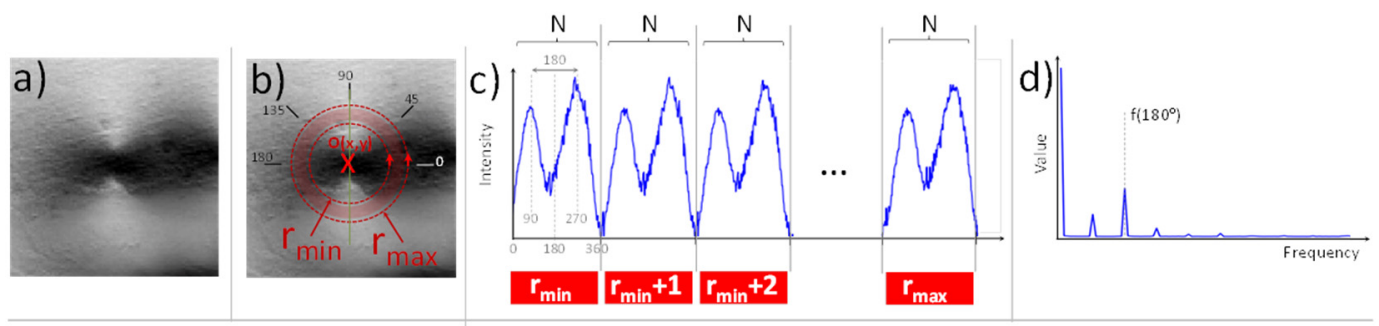

e)

f)

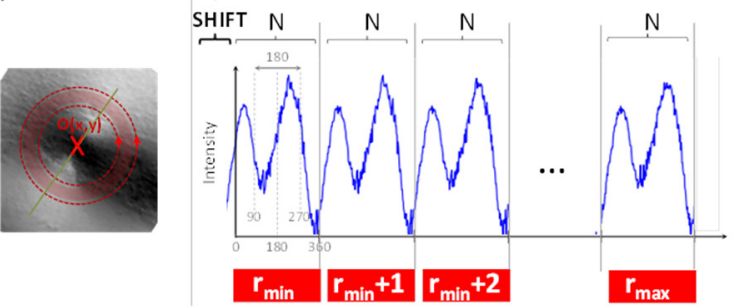

Fig. 4. Description of the method developed for the detection of Butterfly Patterns obtained in Inductive Thermographybased inspections. a) is an example of a butterfly-like structure. b) considers a polar coordinate system where the point $O(x, y)$ is the center of rotation of the butterfly pattern: any representation of the pixel values of the points that lay within the perimeter of a circle with radius between $r_{\min }$ and $r_{\max }$ will show two peaks. $c$ ) If pixel values for different radius are plotted one after the other, the obtained representation will show a periodical pattern with period $180^{\circ}$. d) The amplitude spectrum of the FFT of the obtained signal will show a peak at $180^{\circ}$. e) The same butterfly-like pattern but with a different orientation. f) The obtained signal will be the same as that of c) but shifted due to its different orientation (green lines indicate the orientation of the pattern).

In order to apply this method in an automatic manner, the developed algorithm has been divided into the following four steps (see also Fig. 4 for a graphical description):

1) the thermogram is FFT transformed and its phase is extracted,

2) the developed pDFT filter is applied to the obtained image to identify possible regions in the phase image with rotational symmetry. This filter is equivalent to apply the FFT to each of the signals extracted at each pixels of the image.

3) since the optimum interval [rmin,rmax] for each of the pixels is not known beforehand, both parameters have to be adjusted for each of the pixels. This is done by convolving the phase image with a set of pDFT filters (each for different rmin and rmax values) and identifying the best result for each pixel among the convolved results.

4) the pDFT filter retrieves two results, one for the real part and the other for the imaginary part. In the FFT, the real and imaginary parts can be used to determine the time shift of the input signal at a specific frequency value. In a similar way, the real and imaginary parts of the pDFT can be used to derive the orientation of the detected butterfly-like patterns, i.e. in each pixel, the time shift in this case corresponds to a rotation for the signal extracted for each radius. 
This method has been used to obtain the rough estimate of the radius and the angle of the detected defects shown in the results section.

\section{Results}

This section deals with all the experimental work performed and the applied posterior processing for automatic defect detection. First a brief explanation of the employed parameters is given. Next the importance of the inductor configuration is highlighted and how its orientation affects to the heating, and therefore to the obtained thermograms. Finally, all the thermographic measurements performed are shown together with the corresponding macrograph of the inspected area and the results of the automatic defect detection.

\subsection{Induction Excitation Configuration Optimization}

In order to stablish the optimal parameters for the thermographic measurements, several experiments have been performed on both EBW and TIG samples.

In the case of the EBW samples, Area 1 of Sample 2 (see Fig. 5) has been chosen for this task. The initially considered values for the induction frequency are in the $20-40 \mathrm{kHz}$ range and between 50 and 500 ms for the pulse duration. All these measurements have been performed with inductor I1 (recall section 3, Fig. 2(a)).

Sample 2, Area 1

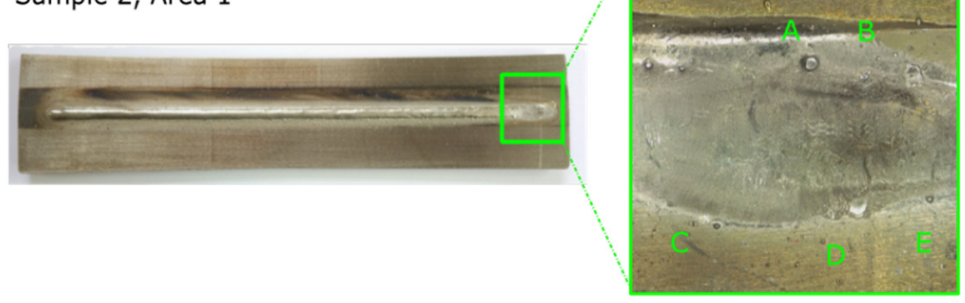

Fig. 5. Selected areas and cracks in Sample 2 (EBW sample) for the optimization of the induction excitation parameters.

After the measurements FFT has been applied to all the recordings considering different frame intervals. As it was mentioned in the previous section, this is a crucial factor in order to obtain the sharpest phase to detect the butterfly shape of the cracks in the posterior detection algorithm application. For the optimal pulses the whole heating and half the cooling has been decided to be the best range. On the one hand, heating contains almost all the butterfly shape effect. On the other hand, considering a larger number of frames in the cooling has been shown to be negative 'saturating' the image and making difficult to distinguish patterns of cracks that are close to each other.

For EBW samples best results have been obtained for pulses ranging between $100 \mathrm{~ms}$ and $200 \mathrm{~ms}$. As for the induction frequency, the heating produced at $20 \mathrm{kHz}$, which is the highest, has been concluded to be the optimal. Integration time has been set to $700 \mu \mathrm{s}$ in all measurements.

In the case of TIG samples, which have been inspected with inductor I2, the considered optimal pulses also range from $100 \mathrm{~ms}$ and $200 \mathrm{~ms}$. However, the optimal induction frequency range is wider: $10 \mathrm{kHz}$ to $35 \mathrm{kHz}$. The results are worse than with inductor I 1 since this one is not still completely optimized and the contact with the surface of the sample is difficulted by its curvature.

\subsection{Inductor Orientation Importance on the Butterfly Defect Shape}

The inductors considered in the present work for the thermal excitation of the samples are orientation dependant. This means that the heating of a crack will be different depending on the relative orientation of the crack $(\alpha)$ considered and the inductor $(\beta)$. What is more, this affects the butterfly shape obtained after applying the FFT to the thermal sequence. Figure Fig. 6 shows a scheme of the prepared setup for the inspection of Samples 4 and 6 . In order to see the effect of the orientation of the inductor 12 when detecting cracks, it has been placed at two different orientations: $\beta=0$ and the optimal. In this case the optimal configuration occurs when the angle $\beta$ of the inductor coincides with the perpendicular of the crack. In other words, the best configuration corresponds to the condition when the magnetic field is parallel to the 
crack to be inspected and the induced eddy currents occur at angle $\beta$. Results of the thermographic inspection for Samples 4 and 6 are shown on Fig. 7 and Fig. 8 respectively. Clearly, when the orientation is optimized, the butterfly shape is much more symmetric and clear, which is invaluable for the application of the detection algorithm in the posterior step.
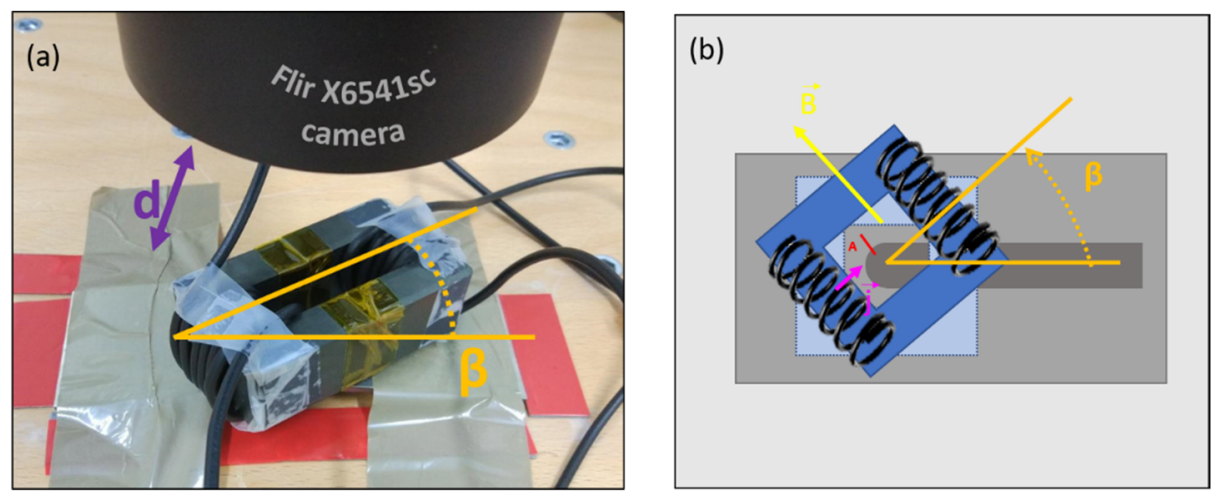

Fig. 6. The figure shows the optimal setup for the detection of crack $A$. As can be seen inductor 12 has been employed positioned so that the angle with respect to the horizontal is $\beta$ approximately. This is in fact the direction of the induced eddy currents, which will hit the crack completely since its angle is at $\alpha=\beta+90$, maximizing the thermal excitation and providing a symmetric butterfly shape after the application of the FFT to the thermal recording.
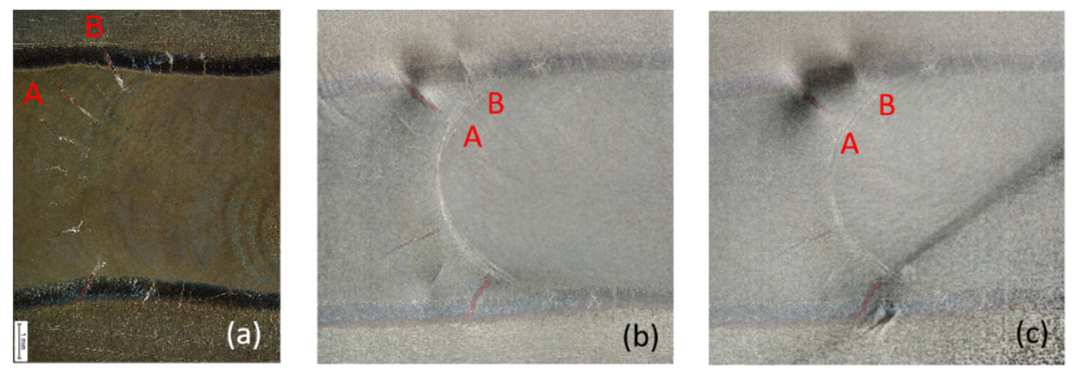

Fig. 7. (a) Sample 4, where crack $A$ and $B$ have been highlighted. (b) and (c) correspond to the thermographic results with $\beta=0$ and $\beta=40^{\circ}$ respectively superposed with the macro. Clearly, the positioning of the inductor is essential to obtain a symmetric butterfly shape, with the maximums at the two edges of the crack.
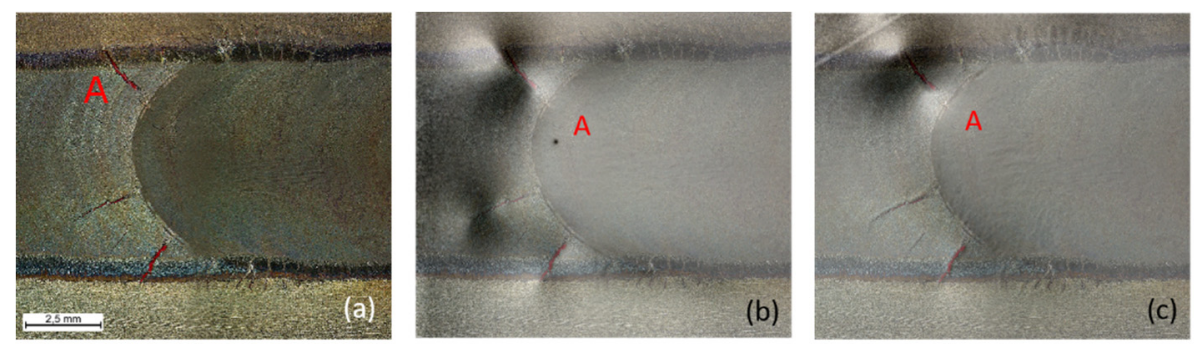

Fig. 8. (a) Sample 6, where crack $A$ has been highlighted. (c) and (d) correspond to the thermographic results with $\alpha=0$ and $\alpha=45^{\circ}$ respectively superposed with the macro.

The effect of the orientation has already been solved in some cases for the inspection of small components [11], manufacturing inductors so that the excitation is independent of the relative orientation with the cracks at the sample to be inspected. Anyway, in the next section it will be seen that even when the orientation is not optimal, the detection algorithm also works. 


\subsection{Automatic Defect Detection Algorithm Results}

In this section the results of the application of the detection algorithm to all the inspected samples are shown. Table 1 and Table 2 collect all the work performed in this research. Each column of the tables represents a different stage of the inspection process. The first column gives the details of the cracks present on each sample. The second column corresponds to the application of the FFT to the thermographic recording. It is at the third column where the first results of the detection algorithm are shown. In particular in this column the algorithm already marks the defects it has found and gives an estimation of the radius of the defect and its orientation. Finally, the fourth and last column corresponds to the obtained complete detection.

The results show that the detection algorithm must be further adjusted, since it still fails in the detection of some defects. In Sample 1 crack $E$ is not detected for example. Furthermore, in some cases it still gives false positives, as happens in Sample 2, where it finds two extra cracks.

As for the capabilities of the algorithm, one of the first things to note is that when the inductor is not located optimally, as was discussed on the previous section, detection still occurs. This can be observed in crack F of Sample 2 , improving of course the result when orientation is turned to $\beta=60^{\circ}$.

Furthermore, a detailed look can be taken to the radius and angle calculations of the algorithm. For this task, crack length and angle have been measured from the macros captured, compiling all the information in Table 3. Note that the calculation of crack length is not straightforward, since in the macro sometimes it cannot be distinguished a crack from a scratch. Furthermore, since for the algorithm the crack is straight, this assumption has also been made when making the measurements in the macros. In the same way, the mean angle of the crack has been considered when measuring its inclination with respect to the horizontal $(\alpha)$. The correlation between the experimental radius and the computed values is still not clear. However, it must be said that the angle correlation is good. This can be checked visually in Fig. 9. 
Table 1. Summary of the results obtained for each inspected piece. The first column indicates the cracks present at each sample, the second column shows the butterfly shape after the FFT has been applied. Third and fourth columns are the result of the application of the detection algorithm: the third one gives an estimation of the size of the defects and their radius. The fourth one is detection of the defects itself.

\begin{tabular}{|c|c|c|c|}
\hline Defects & Thermogram + FFT Phase & $\begin{array}{l}\text { Detection Algorithm pFFT } \\
\text { (Radius) }\end{array}$ & Detection Algorithm (pFFT) \\
\hline \multicolumn{4}{|c|}{$\begin{array}{l}\text { Sample 1: Cracks A, B, C, D, E } \\
\text { Camera Parameters: } F \text { lir } x 6541 \mathrm{sc} \text { with extension ring, } d=120 \mathrm{~mm} \text {, frate }=300 \mathrm{fr} / \mathrm{s}, \mathrm{t}_{\mathrm{rec}}=1 \mathrm{~s} \\
\text { Induction Parameters: } \mathrm{f}_{\text {ind }}=20 \mathrm{kHz}, \mathrm{PWD}=100 \%, \mathrm{t}=300 \mathrm{~ms} \text {, Inductor: } I 1, \beta=0 \mathrm{o}, \\
\text { Processing parameters: } F \mathrm{FT}_{\mathrm{f}}=6.122 \mathrm{~Hz}\end{array}$} \\
\hline $\begin{array}{l}A B \\
\text { B. } \mathrm{B} \\
\end{array}$ & A & (8) & 8 \\
\hline \multicolumn{4}{|c|}{$\begin{array}{l}\text { Sample 2: A, B, C, D, E } \\
\text { Camera Parameters: Flir } x 6541 \mathrm{sc} \text { with extension ring, } d=120 \mathrm{~mm} \text {, frate }=300 \mathrm{fr} / \mathrm{s}, \mathrm{t}_{\mathrm{rec}}=1 \mathrm{~s} \\
\text { Induction Parameters: } \mathrm{f}_{\text {ind }}=20 \mathrm{kHz}, \mathrm{PWD}=100 \%, \mathrm{t}=200 \mathrm{~ms} \text {, Inductor: } 11, \beta=00 \text {, } \\
\text { Processing parameters: } \mathrm{FFT}_{\mathrm{f}}=3.896 \mathrm{~Hz}\end{array}$} \\
\hline 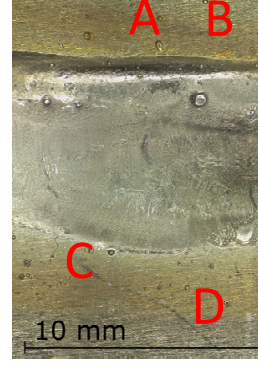 & $\mathrm{D} \cdot \mathrm{E}$ & * ${ }^{A} \mathrm{D}_{+} \mathrm{AT}^{\mathrm{B}}$ & D. \\
\hline \multicolumn{4}{|c|}{$\begin{array}{l}\text { Sample 2: D, E, F } \\
\text { Camera Parameters: Flir } x 6541 \mathrm{sc} \text { with extension ring, } \mathrm{d}=110 \mathrm{~mm} \text {, frate }=300 \mathrm{fr} / \mathrm{s}, \mathrm{t}_{\mathrm{rec}}=1 \mathrm{~s} \\
\text { Induction Parameters: } \mathrm{f}_{\text {ind }}=20 \mathrm{kHz}, \mathrm{PWD}=75 \%, \mathrm{t}=200 \mathrm{~ms} \text {, Inductor: } 11, \beta=-60 \mathrm{O} \text {, } \\
\text { Processing parameters: } \mathrm{FFT}_{\mathrm{f}}=3.896 \mathrm{~Hz}\end{array}$} \\
\hline$x^{0} \cdot 4$ & $\mathrm{E}_{\mathrm{F}}$ & D E F & E F \\
\hline
\end{tabular}


Table 2. Continuation of Table 1. The first column indicates the cracks present at each sample, the second column shows the butterfly shape after the FFT has been applied. Third and fourth columns are the result of the application of the detection algorithm: the third one gives an estimation of the size of the defects and their radius. The fourth one is detection of the defects itself.

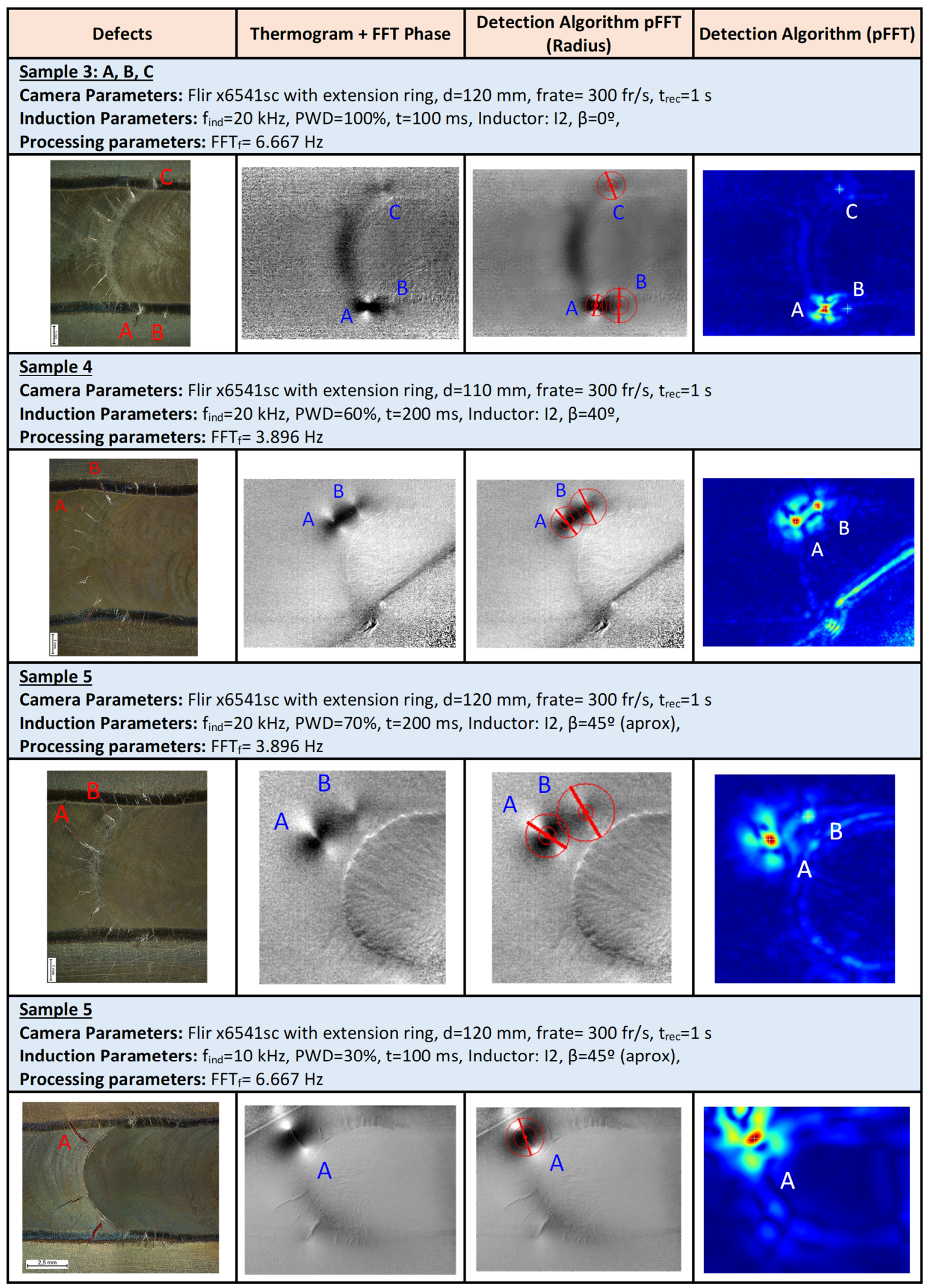


Table 3. Table summarizing the experimental and detection algorithm results corresponding to the length of the inspected cracks and their angle for several samples.

\begin{tabular}{|c|c|c|c|c|c|c|c|}
\hline Sample & $\begin{array}{c}\text { Welding } \\
\text { Type }\end{array}$ & Crack & $\begin{array}{c}\text { Experimental } \\
\text { Crack } \\
\text { Size }(\mathrm{mm})\end{array}$ & $\begin{array}{c}\text { Calculated mean } \\
\text { Crack Size (pixels) } \\
\text { TH=0.95 }\end{array}$ & $\begin{array}{c}\text { Calculated mean } \\
\text { Crack Size }(\mathrm{mm}) \\
\mathrm{TH}=0.95\end{array}$ & $\begin{array}{c}\text { Angle (o) } \\
\text { (Experimental Measurement) }\end{array}$ & $\begin{array}{c}\text { Angle (o) } \\
\text { (Algorithm Result) }\end{array}$ \\
\hline \multirow{4}{*}{1} & \multirow{4}{*}{ EBW } & A & 1.90 & 11.5 & 0.55 & 94.9 & 86.7 \\
\hline & & $B$ & 1.36 & 8.5 & 0.41 & 89.3 & 71.0 \\
\hline & & C & 1.07 & 12.5 & 0.60 & 77.6 & 76.9 \\
\hline & & $D$ & 1.60 & 9.5 & 0.46 & 86.4 & 86.8 \\
\hline \multirow{6}{*}{2} & \multirow{6}{*}{ EBW } & A & 0.72 & 20.5 & 0.98 & 94.2 & 94.4 \\
\hline & & $B$ & 0.66 & 16.5 & 0.79 & 83.8 & 92.5 \\
\hline & & C & 0.90 & $\begin{array}{l}13.5 \\
\end{array}$ & 0.65 & 77.5 & 76.8 \\
\hline & & D & 0.93 & 12.5 & 0.60 & 105.0 & 108.0 \\
\hline & & $E$ & 0.94 & 111.0 & 0.53 & $\begin{array}{l}78.4 \\
\end{array}$ & 76.2 \\
\hline & & $F$ & 1.57 & 19.5 & 0.94 & 127.3 & 105.9 \\
\hline 3 & TIG & $B$ & 1.07 & 20.0 & 0.96 & 83.2 & 89.6 \\
\hline \multirow{2}{*}{4} & \multirow{2}{*}{ TIG } & A & 1.43 & 26.5 & 1.27 & 118.1 & 120.8 \\
\hline & & $B$ & 2.13 & 14.5 & 0.70 & 140.2 & 146.3 \\
\hline
\end{tabular}

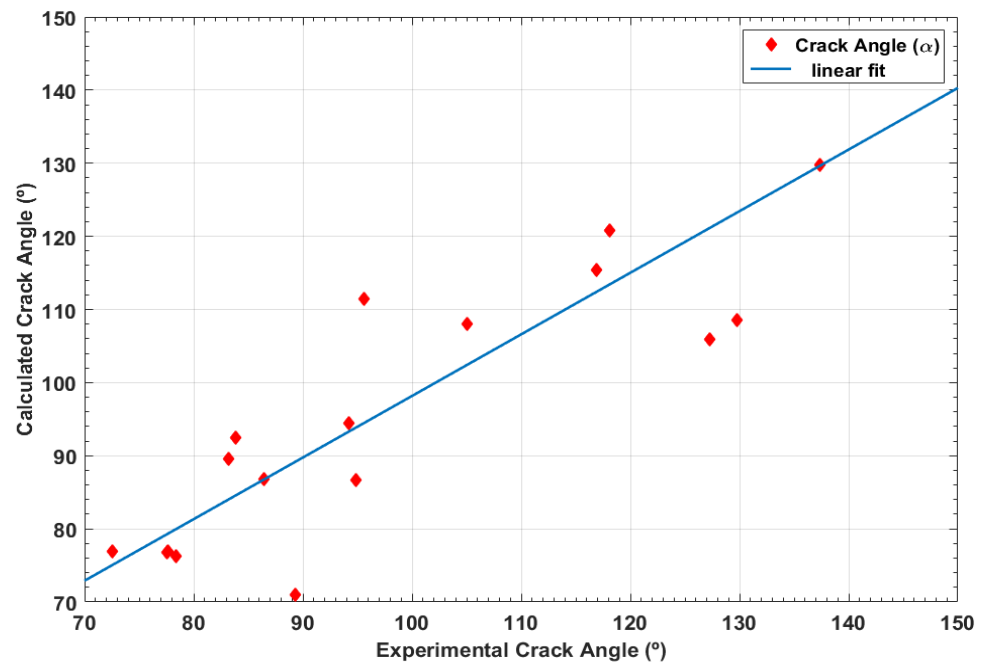

Fig. 9. Correlation between the experimentally measured crack angle and the computed one by the developed crack detection algorithm.

\section{Conclusions and Future Work}

In this paper pulsed induction thermography has been applied to Inconel $718 \mathrm{EBW}$ and TIG samples in order to detect surface cracks. Induction parameters have been optimized for an easier later processing. As for the processing, in a first step FFT has been applied, keeping just the heating information and only part of the cooling curve in order to better visualize the butterfly shape appearing at the cracks. This conclusion is still preliminary and should be checked if it can be generalized for all cracks in Inconel 718.

The main contribution of the paper arises with the application of the developed Crack Detection Algorithm to the obtained FFT images. It finds the rotational symmetry of the 'Butterfly-like Patterns' to convert them by using a polar Discrete Fourier Transform into 2D Gaussian like distributions that allow the identification of cracks.

The algorithm works pretty well even though it still fails sometimes due to possible faint butterfly shapes that can be overlooked. Sometimes it still detects also certain periodicity in the sample which is not necessarily associated to a butterfly shape. 
Apart from these points still under improvement, it is robust enough to even capture the defects when the inductor is not optimally situated with respect to the crack, i.e. when the butterfly shape is distorted. Furthermore, the algorithm is capable of estimating both crack length and its orientation, even if this subject is still under research.

Currently the work is being centred in the optimization of the algorithm in order to avoid the mentioned false positives. In this sense, also experimental work is being performed: on one hand to improve the measurements and on the other hand to improve the inductors. Having crack orientation independent inductors would make easier the detection, since FFT images would be cleaner. Although there already exists this type of inductors [11], which are designed for the inspection small samples, they are useless for the inspection of the real final big sized Inconel 718 components considered here.

\section{REFERENCES}

[1] Christian Srajbr, Alexander Dillenz, and Klaus Bräutigam. Crack detection at aluminum fuselages by induction excited thermography. In 4th International Symposium on NDT in Aerospace 2012 - Th.3.A.3, 2012.

[2] Christian Srajbr. Induction excited thermography in industrial applications. 19th World Conference on NonDestructive Testing 2016, 2016.

[3] Udo Netzelmann, Günter Walle, Sergey Lugin, Andreas Ehlen, Steffen Bessert, and Bernd Valeske. Induction thermography: principle, applications and first steps towards standardisation. Quantitative InfraRed Thermography Journal, 13(2):170-181, 2016.

[4] A. Averbuch, R.R. Coifman, D.L. Donoho, M. Elad, and M. Israeli. Fast and accurate polar fourier transform. Applied and Computational Harmonic Analysis, 21(2):145 - 167, 2006.

[5] Markus Fenn, Stefan Kunis, and Daniel Potts. On the computation of the polar fft. Applied and Computational Harmonic Analysis, 22(2):257 - 263, 2007.

[6] Rubén Usamentiaga, Pablo Venegas, Jon Guerediaga, Laura Vega, Julio Molleda, and Francisco G. Bulnes. Infrared thermography for temperature measurement and non-destructive testing. Sensors, 14(7):12305-12348, 2014.

[7] Beate Oswald-Tranta and Mario Sorger. Localizing surface cracks with inductive thermographical inspection: from measurement to image processing. Quantitative InfraRed Thermography Journal, 8(2):149-164, 2011.

[8] Beata Oswald-Tranta. Thermoinductive investigations of magnetic materials for surface cracks. Quantitative InfraRed Thermography Journal, 1(1):33-46, 2004.

[9] Oswald-Tranta B. and G. Wally. Thermo-inductive surface crack detection in metallic parts. 2006.

[10] Edevis Gmbh - https://www.edevis.com.

[11] Oswald-Tranta. Investigations for determining surface crack depth with inductive thermography. In 19th World Conference on Non-Destructive Testing 2016, 2016.

[12] Benjamin David Weekes. Investigation of Infrared Thermography NDE Techniques for Use in Power Station Enviroments. PhD thesis, Imperial College London, 2011. 